\title{
An assessment of the integrated nutrition programme for malnourished children aged six months to five years at primary healthcare facilities in Mangaung, Free State, South Africa
}

\author{
Hanneke Brits ${ }^{a *}$ (D), Gina Joubert ${ }^{b}$ iD, Keshia Eymanc, Rosie De Vinkc, Katleho Lesaoanac, Sello Makhethac and Katiso Moeketsic \\ ${ }^{a}$ Faculty of Health Sciences, Department of Family Medicine, University of the Free State, Bloemfontein, South Africa \\ ${ }^{b}$ Faculty of Health Sciences, Department of Biostatistics, University of the Free State, Bloemfontein, South Africa \\ 'Faculty of Health Sciences, School of Medicine, University of the Free State, Bloemfontein, South Africa \\ *Corresponding author, email: britsh@ufs.ac.za
}

Background: According to the World Health Organization (WHO), malnutrition can be linked to $45 \%$ of deaths in children under the age of five years. The Integrated Nutrition Programme (INP) was introduced in 1994 to address malnutrition in South Africa. There had been no systematic evaluation of how well clinics perform regarding nutritional services. Malnutrition rates worsened in the Free State from 3.9\% in 2009 to $10.7 \%$ in 2013. This study aimed to assess the effectiveness of the INP Supplementary Feeding Programme in primary healthcare facilities in the Mangaung University of the Free State Community Partnership Project, known as MUCPP, catchment area of Bloemfontein, Free State, in children aged six months to five years.

Methods: This was a retrospective, descriptive cohort study. All children between six months and five years entering the feeding scheme between July 2014 and June 2015 at the MUCPP, which is the hub of the feeding scheme, and three primary healthcare clinics were included. Data were collected from September to November 2015 from the INP registers and captured on dataextraction forms.

Results: In total, 730 children were included in the study, $38.8 \%$ at risk for malnutrition and $61.2 \%$ malnourished. Most of the children (80.7\%) stopped attending before change was seen. Only $14.1 \%$ exited the INP successfully with a normal weight for age and $3.0 \%$ deteriorated despite nutrition interventions. Of all children with one or more follow-up visits, 50.4\% (119/236) improved from severe malnutrition to underweight or exited at target weight.

Conclusions: The follow-up of the children and the monitoring and implementation of the INP are inadequate. There is some evidence that children who attend the INP regularly benefit from the programme.

Keywords: children, Free State, malnutrition assessment, nutrition programme, South Africa, supplementary feeding

\section{Introduction}

According to the World Health Organization (WHO), malnutrition can be linked to $45 \%$ of deaths in children under the age of five years. ' There are many definitions of child malnutrition, but Ge and Chang summarised it as:

'A pathological state resulting from inadequate nutrition, including undernutrition (protein-energy malnutrition) due to insufficient intake of energy and other nutrients; overnutrition (overweight and obesity) due to excessive consumption of energy and other nutrients; deficiency diseases due to insufficient intake of one or more specific nutrients such as vitamins or minerals.'2

Anthropometric measurements based on gender and age, currently z-scores, are used to assess the nutritional status in children. ${ }^{3}$

Poverty and inequity are major contributors to malnutrition, both of which are rife in South Africa. ${ }^{4-7}$ In populations where there is a high prevalence of malnutrition, a typical meal contains mostly starch-rich products, such as maize, as this is more affordable. There is often a limited intake of fruits, vegetables, legumes, and few or no animal products in the diet. ${ }^{8}$ These diets have insufficient nutrients and minerals, which may lead to malnutrition. It is especially important for infants and young children to receive proper nutrition as they are more vulnerable to undernutrition and subsequent impaired growth, due to their high nutritional needs. ${ }^{9}$ The first 1000 days of life are considered the most important for nutrition and, if malnutrition is not addressed by the age of five years, the child is unlikely to reach his/her full potential in life. ${ }^{10,11}$

The Integrated Nutrition Programme (INP) was introduced in South Africa in 1994 to address malnutrition. ${ }^{12}$ Numerous feeding schemes fall under this programme, such as the Primary School Nutrition Programme (PSNP), community programmes and food parcels. The INP is a multi-sectorial programme, which includes the Departments of Health, Social Development and Agriculture. In 2010, the Nutrition Supplementation Programme (part of the INP) was renamed the Nutrition Therapeutic Programme (NTP) to emphasise that it was not just a social welfare programme but a therapeutic measure to address malnutrition. ${ }^{12}$

The purpose of the INP is to guide health workers on health promotion, supplementary feeding to those who are malnourished or at risk of becoming malnourished, rehabilitation of the malnourished individuals, and the continuous monitoring and evaluation of the INP. Depending on their age, children receive different supplements. Infants between six and 12 months receive formula milk and infant cereal, and from one year onwards children receive enriched maize meal (EMM), lactose-free energy drinks and ready-to-use therapeutic food (RUTF) according to government tenders. ${ }^{9}$ 
According to Iversen et al., ${ }^{12}$ there is an apparent lack of improvement shown by several growth indicators, despite the INP. They noted there had been no systematic evaluation of how well clinics perform in terms of nutritional services, hence it is difficult to describe how well the INP functions. Malnutrition rates actually worsened in the Free State from 3.9\% in 2009 to $10.7 \%$ in $2013 . .^{13}$ The fact that the INP has not been sufficiently evaluated motivated the authors to investigate the INP in their region.

The South African Supplementary Feeding Guidelines for at Risk and Malnourished Children and Adults make provision to assess and classify patients. ${ }^{9}$ Depending on the assessment, children will be classified as severe acute malnutrition (SAM), moderate acute malnutrition (MAM), conditions with increased nutritional needs, or growing appropriately. Children with SAM must be referred to a clinic or hospital for further assessment and management by a doctor, while children with MAM and those with increased nutritional needs (at risk for malnutrition) must receive supplementary food (Plan B) at the clinic. Children growing appropriately must be counselled and followed up. The Implementation Guidelines for Nutritional Interventions at Health Facilities $^{14}$ indicate the entry criteria according to the Nutritional Risk Score (NRS), follow-up, and exit criteria for children receiving supplemental food at the clinics. An NRS of 4-5 will put a child in the 'at risk' category and an NRS of $\geq 6$ in the 'malnourished' category. Both these groups qualify for therapeutic supplements (Plan B). A child will qualify to exit the programme if the NRS is $<4$. However, if the NRS does not improve over a period of six months, the child must also be referred for a doctor's assessment. ${ }^{14}$

The majority of identified malnourished children in Bloemfontein attend the MUCPP (Mangaung University of the Free State Community Partnership Project) Community Health Centre. The MUCPP is a public organisation providing primary healthcare to the community and is the hub of the feeding scheme with a dietitian in charge of the programme. Surrounding clinics receive supplements, such as fortified porridge and milkshakes, and support from MUCPP.

\section{Aim and objectives}

The aim of this study was to assess the effectiveness of the INP supplementary feeding programme (Plan B) in primary healthcare facilities in the MUCPP draining area of Bloemfontein, in children aged six months to five years. Effectiveness was determined by the percentage of children who successfully exited the INP.

\section{Objectives of the study were to determine:}

- the percentage of children who exited the feeding programme successfully;

- the percentage of children (for both 'at risk' and 'malnourished' children, separately)

(i) who did not complete the feeding programme before completion;

(ii) where the nutritional status deteriorated while on the feeding programme; and

(iii) who improved, but did not meet the exit criteria of a NSR $<4$

\section{Method}

\section{Study design, population and sampling}

This was a retrospective, descriptive cohort study. MUCPP and the four smaller clinics (Heidedal, Kagisanong, Thusanong and Batho) served by the centre were purposefully selected for the study. These facilities use a similar malnutrition recording system and share the same dietitian. One clinic (Batho) was closed during the study period for renovations, and the patients were transferred to the facility in Heidedal. These patients are all reflected under Heidedal. All children between the ages of six months and five years enrolled in the feeding scheme between July 2014 and June 2015 at these facilities were included in the study. On admission to the INP supplementary feeding programme, all children were assessed, received supplemental feeding and were then entered into the register. There were no exclusion criteria.

\section{Data collection}

The performance of the health facilities was assessed using the South African INP guidelines that were implemented in 2013. ${ }^{14}$

Data were collected from September to November 2015 from the INP registers at each clinic and captured on a data-extraction form. Information included demographic data, enrolment date, follow-up dates, weight, underlying conditions, type of supplementation provided, comments and outcome. To determine their nutritional status, weight-for-age was categorised for each child per visit using the WHO Child Growth Standards (Annexure $\mathrm{G}$ of the implementation guidelines). ${ }^{14}$

The NRS was not recorded, but it was possible to calculate the score and group the children as either 'at risk' or 'malnourished' with the data available.

\section{Pilot study}

A pilot study was conducted during July 2015 on 10 patients from MUCPP clinic. The study showed that the mid-upper arm circumference (MUAC) was not recorded for any of the children; however, this measurement was not necessary, as the weight and age for the children were available. The data-extraction form used in the main study was adjusted to include all the data available in the INP registers according to the register format to make data collection easier. All 10 patients from the pilot study were included in the main study.

\section{Data analysis}

Data from the data forms were entered into an Excel $^{\oplus}$ (Microsoft Corp, Redmond, WA, USA) spreadsheet by the researchers. Data were analysed by the Department of Biostatistics, Faculty of Health Sciences, University of the Free State (UFS), using SAS/ STAT software, Version 9.3 (@2010, SAS Institute, Cary, IN, USA). Results were summarised by frequencies and percentages in tables.

\section{Ethical considerations}

The researchers obtained approval from the Free State Health Department, as well as the Ethics Committee of the Faculty of Health Sciences, UFS, to perform the study (STUD NR 35/2015). Patient names were not recorded on data-extraction forms, but were checked before data entry to ensure that no duplication occurred and that all follow-up visits were recorded. An identification number was allocated to each patient. 
Table 1: Entry nutritional status of children receiving supplemental food at the clinics

\begin{tabular}{lc}
\hline Category & $\boldsymbol{n}(\%)$ \\
\hline Normal weight (at risk for malnutrition) & $283(38.8)$ \\
Underweight & $178(24.4)$ \\
Severely underweight & $269(36.8)$ \\
Total & $730(100.0)$ \\
\hline
\end{tabular}

Table 2: Number of clinic visits $(n=730)$

\begin{tabular}{lcc}
\hline Number of visits & $\boldsymbol{n}(\%)$ & Cumulative \% \\
\hline Initial visit only & $494(67.7)$ & 67.7 \\
\hline Initial visit + 1 follow-up visit & $124(17.0)$ & 84.7 \\
\hline Initial visit + 2 follow-up visits & $53(7.3)$ & 92.0 \\
\hline Initial visit + 3 follow-up visits & $31(4.3)$ & 96.2 \\
\hline Initial visit + 4 follow-up visits & $21(2.7)$ & 89.9 \\
\hline Initial visit + 5 follow-up visits & $7(1.0)$ & 99.9 \\
\hline Initial visit + 6 follow-up visits & $1(0.1)$ & 100.0 \\
\hline
\end{tabular}

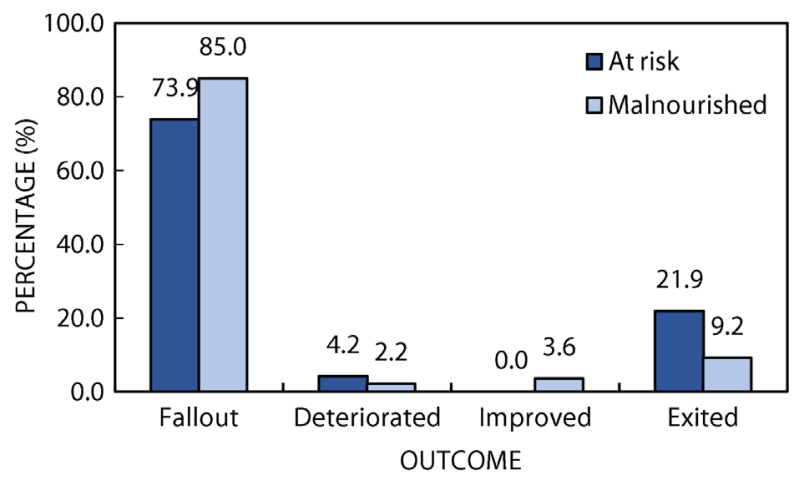

Figure 1: Outcome of children who entered the INP programme malnourished and at risk $(n=730)$.

\section{Results}

Data were collected for all 730 children in the INP programme during the study period. Gender distribution was as follows: 349 (48.0\%) females and 378 (52.0\%) males (gender of three children was not indicated). The distribution between the clinics was skewed, with $82.3 \%(n=601)$ from MUCPP, $12.3 \%(n=90)$ from Heidedal, 3.3\% $(n=24)$ from Kagisanong and 2.1\% $(n=15)$ from Thusanong clinic.

Almost $40 \%(38.8 \%, n=283)$ of the children fell in the 'at risk' category and $61.2 \%(n=447)$ were malnourished. In Table 1 , the entry nutritional status of the children, according to WHO weight-for-age criteria, is displayed.

The majority of children (67.7\%) attended the clinic only for the initial visit. The number of clinic visits varied between one and seven. In Table 2, the number of visits is reflected as a percentage of patients.

All the 'at risk' children started at normal weight for their age. The majority of these children $(73.9 \%, n=209)$ did not return for follow-up visits, $21.9 \%(n=62)$, exited the programme at normal weight and $4.2 \%(n=12)$ deteriorated and became malnourished.

Only $9.2 \%$ of the malnourished children who started with the INP exited the programme at normal weight for age. A nutritional status change from severe malnutrition to underweight was considered an improvement, and a change from underweight to severe malnutrition was considered a deterioration. Figure 1 displays the outcomes of the children in the INP.

Despite the high fallout rate before reaching the target weight, many children improved/exited with follow-up visits. Of all children with one or more follow-up visit, 50.4\% (119/236) improved or exited at target weight. For the 'at risk' children, this percentage was $82.7 \%(62 / 75)$ compared with $35.4 \%$ of malnourished children (57/161). In Figure 2, the percentage of children who deteriorated, improved and exited as well as patients who did not complete (fallout) is depicted per number of follow-up visits.

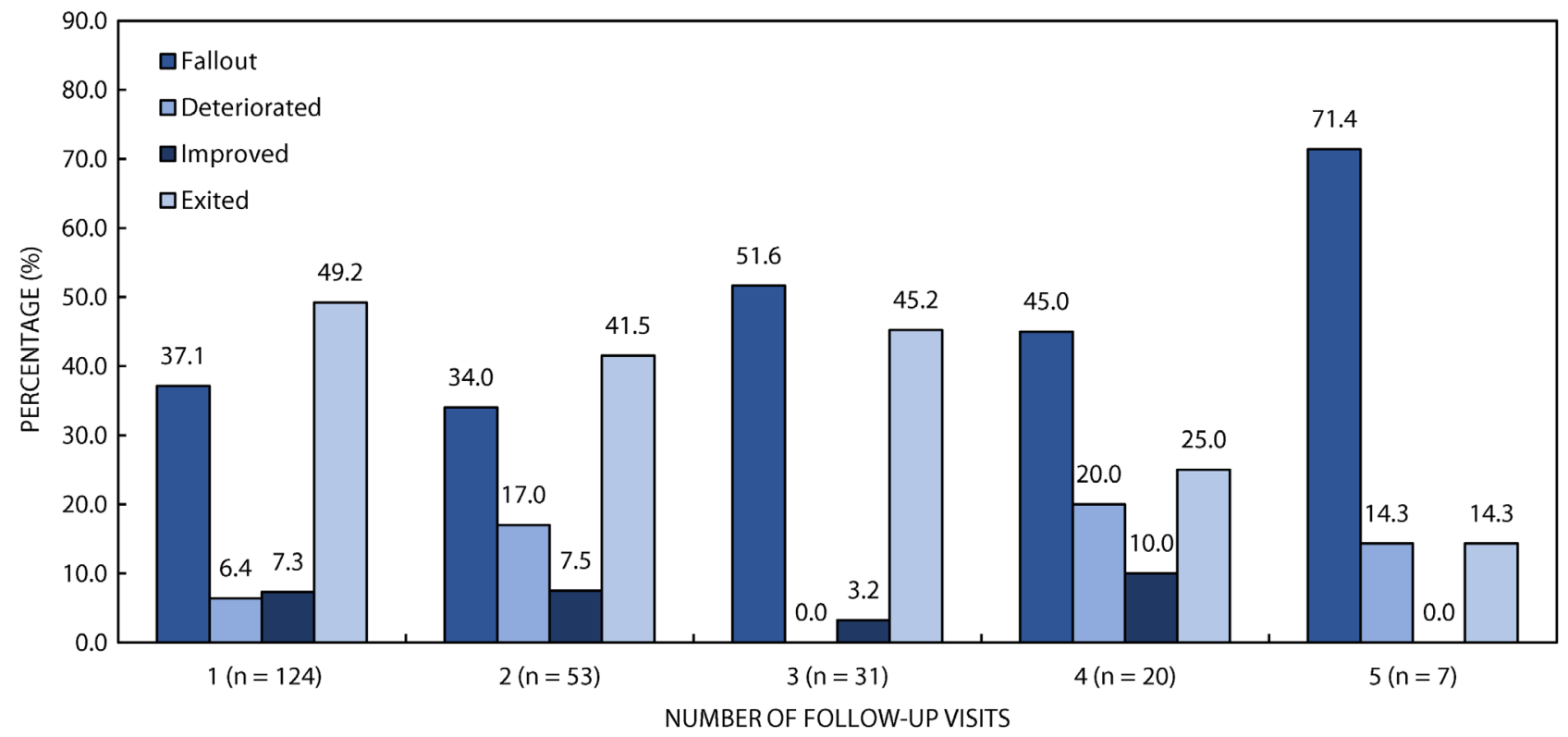

Figure 2: Percentage of children who deteriorated, improved and exited, as well as fallout patients per number of follow-up visits. 
Per clinic, the percentage of children exiting the programme ranged from $5.6 \%$ to $15.8 \%$.

\section{Discussion}

During data collection, it became clear that primary healthcare clinics in Mangaung do not follow the INP guidelines strictly. The MUAC was not measured in any of the children and the NRS was not recorded for some of the patients. However, it was possible to calculate the NRS from the data available. In a South African study where MUAC and weight for height $(W / H)$ were compared for malnourished children, the conclusion was that MUAC is a good screening tool, but that $\mathrm{W} / \mathrm{H}$ was more sensitive and better used by healthcare workers in clinics. ${ }^{15}$

The MUCPP is a community health centre with longer working hours, is easily accessible, and has doctors and a dietitian on site. This can explain why the majority of children were seen there, and not in the smaller clinics.

Only $1.1 \%$ of children completed at least six months on the supplemental feeding programme. Two-thirds attended the programme only once and then never returned. During communication with the dietitian and nursing staff, a few reasons were mentioned for the poor follow-up, which include the long waiting times at clinics, distance to the clinic, unavailability of supplements at the clinic at the time of visit and stigmatisation.

In the 'at risk' category, $4.2 \%$ of children became malnourished (deteriorated) despite attending the feeding programme. In a study in Ethiopia ${ }^{16}$ where at-risk children were followed up, but did not receive supplemental food, $45 \%$ deteriorated despite regular follow-up visits. There is therefore a place for providing supplemental food to at-risk children.

In the malnourished group, only $9.2 \%$ of the children exited the programme successfully at normal weight for age, and a further $3.6 \%$ improved from severe malnutrition to underweight for age. These findings are consistent with the results of the assessment of feeding programmes in South Africa between 1990 and 2010, which showed that the malnutrition intervention programmes in South Africa failed to improve the nutritional status of those targeted. ${ }^{12}$ The fallout of $85 \%$ highlighted a problem with implementation and monitoring of the INP. A 2015 Cochrane review $^{17}$ of 32 studies assessing food supplementation programmes for children aged between three months and five years concluded that these programmes are underperforming in reaching targets, despite evidence that feeding programmes are beneficial to the participants. The main problems with these programmes are implementation and monitoring. ${ }^{17}$

The different clinics were grouped together due to transfer of some patients to Heidedal clinic on closure of Batho clinic and the very small numbers (15 and 24 ) in the two other clinics. However, all the clinics performed poorly on reaching target weights.

\section{Conclusion}

The results of this study showed that $14.1 \%$ of children exited the INP successfully; $21.9 \%$ were in the 'at risk' group and $9.2 \%$ in the malnourished group. However, $80.7 \%$ of children attended the INP only once, or exited before any change was noted.

In the malnourished group, $3.6 \%$ of the children showed improvement from severe malnutrition to underweight. Despite attendance at the INP, the nutritional status of $3.0 \%$ of children deteriorated.
There is evidence that children who attend the INP regularly benefit from the programme; however, the follow-up of the children and the monitoring and implementation of the INP are problematic.

\section{Recommendations}

It is recommended that the reasons why only $9.2 \%$ of all children exited the INP in Mangaung successfully be investigated. This must include (i) obstacles in implementing the INP guidelines correctly, (ii) reasons for the high fallout rate, and (iii) obstacles to the monitoring of the INP.

In order to reduce the fallout rate, the Home-Based Care teams can form an integral part of the INP. During home visits, they can motivate the caregivers to take children back to the clinic, as well as identify obstacles preventing caregivers returning to the clinic.

Acknowledgements - The authors wish to thank Ms T Mulder, medical editor, School of Medicine, UFS, for technical and editorial preparation of the manuscript

Declaration of conflict of interest - The authors report no conflicts of interest.

\section{ORCID}

Hanneke Brits (D) http://orcid.org/0000-0002-5183-5345

Gina Joubert (D) http://orcid.org/0000-0002-3728-6925

\section{References}

1. World Health Organization. Children: reducing mortality. Geneva: Author; 2016 September. [cited 2016 December 27]. Available from: http://www.who.int/mediacentre/factsheets/fs178/en/

2. Ge KY, Chang SY. Definition and measurement of child malnutrition. Biomed Environ Sci. 2001:14(4):283-91.

3. De Onis M, Blössner M. WHO global database on child growth and malnutrition. Geneva: World Health Organization; 1997:49-50. [cited 2016 December 27]. Available from: http://apps.who.int/iris/ bitstream/10665/63750/1/WHO_NUT_97.4.pdf

4. Van de Poel E, Hosseinpoor AR, Speybroeck N, et al. Socioeconomic inequality in malnutrition in developing countries. Bull World Health Organ. 2008;86(4):241-320. doi:10.2471/BLT.07.044800.

5. Benatar SR. The challenges of health disparities in South Africa. S Afr Med J. 2013;103(3):154-5. doi:10.7196/SAMJ.6622.

6. Chitiga M. Income inequality and limitations of the gini index: the case of South Africa. Pretoria: Human Science Research Council; 2014 [cited 2016 December 27]. Available from: http://www.hsrc.ac.za/en/ review/hsrc-review-november-2014/limitations-of-gini-index

7. De Lannoy A, Swartz S, Lake L, et al., editors. South African Child Gauge 2015. Cape Town: Children's Institute, University of Cape Town; 2015.

8. Michaelsen KF, Hoppe $C$, Roos N, et al. Choice of foods and ingredients for moderately malnourished children 6 months to 5 years of age. Food Nutr Bull. 2009;30(3 Suppl):S343-404 https://doi.org/10.1177/15648265090303S303

9. Department of Health, Republic of South Africa. The South African supplementary feeding guidelines for at risk and malnourished children and adults. 2012. Pretoria: Author.

10. Cusick S, Georgieff MK. The first 1,000 days of life: the brain's window of opportunity. New York, NYUnicef; 2014. [cited 2016 December 27]. Available from: https://www.unicef-irc.org/article/958/

11. May J Why child malnutrition is still a problem in South Africa 22 years into democracy. The Conversation; 2016. [cited 2016 December 27]. Available from: http://theconversation.com/why-child-malnutritionis-still-a-problem-in-south-africa-22-years-into-democracy-60224

12. Iversen $P O$, Marais $D$, du Plessis $C$, Herselman M. Assessing nutrition intervention programmes that addressed malnutrition among young children in South Africa between 1994-2010. Afr J Food, Agri Nutr Develop. 2012;12(2):5928-45. 
13. National Department of Health, South Africa. 2nd Triennial report of the committee on morbidity and mortality in children under 5 years (CoMMiC): Triennium 2011-2013. Pretoria: NDoH; 2014. [cited 2016 December 27]. Available from: http://www.kznhealth.gov.za/ mcwh/2nd-CoMMiC-Triennial-Report-2014.pdf

14. Department of Health, Republic of South Africa. Implementation guidelines for nutrition interventions at health facilities; 2013. [cited 2016 December 27]. Available from: http://www.up.ac.za/media/ shared/62/ZP_Files/implementation_guidelines_for_nutrition_ interventions_at_health.zp70745.pdf

15. Dukhi N, Sartorius B, Taylor M. Mid-upper arm circumference (MUAC) performance versus weight for height in South African children (059 months) with acute malnutrition. South Afr J Clin Nutr. 2017;1(1): 1-6. doi:10.1080/16070658.2016.1255483.
16. James P, Sadler K, Wondafrash M, et al. Children with moderate acute malnutrition with no access to supplementary feeding programmes experience high rates of deterioration and no improvement: results from a prospective cohort study in rural Ethiopia. PLoS ONE. 2016;11(4):e0153530. doi:10.1371/journal.pone.0153530.

17. Kristjansson E, Francis DK, Liberato $S$, et al. Food supplementation for improving the physical and psychosocial health of socioeconomically disadvantaged children aged three months to five years. Cochrane Database Syst Rev. 2015;(3):CD009924. https://doi. org/10.1002/14651858.CD009924.pub2

Received: 23-03-2017 Accepted: 06-06-2017 